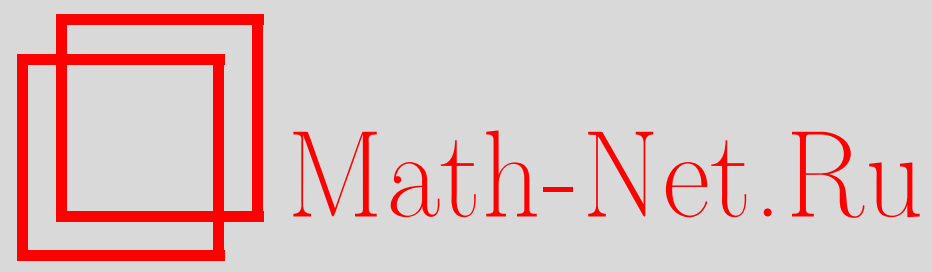

А. Дубицкас, К. Смит, О длине суммы и произведения алгебраических чисел, Матем. заметки, 2005, том 77, выпуск $6,854-860$

DOI: https://doi.org/10.4213/mzm2540

Использование Общероссийского математического портала Math-Net.Ru подразумевает, что вы прочитали и согласны с пользовательским соглашением http://www.mathnet.ru/rus/agreement

Параметры загрузки:

IP : 54.196.121.252

26 апреля 2023 г., 15:23:55

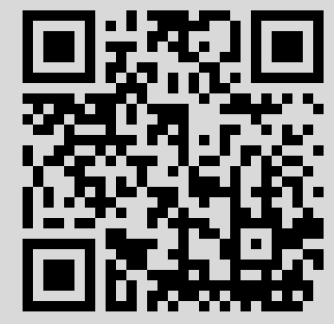




\title{
О ДЛИНЕ СУММЫ И ПРОИЗВЕДЕНИЯ АЛГЕБРАИЧЕСКИХ ЧИСЕЛ
}

\author{
А. Дубицкас, К. Смит
}

В настоящей работе рассматриваются произведения длин алгебраических чисел, сумма или произведение которых является фиксированным алгебраическим числом. Они используются для построения новой функции высоты алгебраических чисел, с помощью которой вводится метрика на множестве всех алгебраических чисел, индуцирующая дискретную топологию.

Библиография: 7 названий.

1. Введение. Пусть $\mathbb{N}, \mathbb{Z}, \mathbb{Q}, \overline{\mathbb{Q}}, \overline{\mathbb{Q}}^{*}$ обозначают соответственно множество натуральных чисел, кольцо целых чисел, поле рациональньх чисел, поле алгебраических чисел и мультипликативную группу алгебраических чисел, отличных от нуля. Через $L(\alpha)$ мы будем обозначать длину алгебраического числа $\alpha$, т.е. сумму модулей коэффициентов его минимального многочлена в $\mathbb{Z}[x]$. На множествевсех многочленов с комплексньми коэффициентами $L(f)$ является очень удобной функцией высоты (сложности многочлена), так как верны неравенства $L(f+g) \leqslant L(f)+L(g)$ и $L(f g) \leqslant L(f) L(g)$. Однако на множестве всех алгебраических чисел $\overline{\mathbb{Q}}$ эти свойства длины $L(\alpha)$ не вьполняются.

В работах [1], [2] рассматривались новые функции высоты алгебраических чисел, основанные на мере Малера и обычной высоте алгебраического числа, используя которые можно построить метрику на некоторых факторгруппах группы $\overline{\mathbb{Q}}^{*}$. Напомним, что мерой Малера алгебраического числа $\alpha$ назьвается произведение старшего коэффициента его минимального многочлена в $\mathbb{Z}[x]$ и всех его корней, по модулю больших единицы. Мера Малера обозначается через $M(\alpha)$, а через $h(\alpha)=(1 / d) \log M(\alpha)$, где $d=\operatorname{deg} \alpha$, мы будем обозначать логарифмическую высоту Вейля числа $\alpha$. Ранее, Шмидт [3] заметил, что $h(\alpha / \beta)$ является метрикой на факторгруппе $\overline{\mathbb{Q}}^{*} / \Omega$, определяющей расстояние между $\alpha \Omega$ и $\beta \Omega$, где $\Omega$ - мультипликативная группа всех корней из единицы. В [1] показано, как на этом же множестве построить метрику, используя так называемую метрическую меру Малера. При этом получаемая топология на $\overline{\mathbb{Q}}^{*} / \Omega$ будет дискретной тогда и только тогда, когда верна гипотеза Лемера. Позднее эта же идея была применена и для построения метрической высоты, основанной на обычной высоте алгебраического числа (см. [2], [4]).

Целью настоящей работы является построение метрики с использованием длины $L$. Это построение кажется наиболееестественным, так как расстояние будет введено на $\overline{\mathbb{Q}}$,

Работа первого автора вьполнена при частичной поддержке Литовского фонда студий и науки. 
а не на какой-нибудь факторгрупше $\overline{\mathbb{Q}}^{*}$. Действительно, при любом $\alpha \in \overline{\mathbb{Q}}$ определим $\mathscr{L}(\alpha)$ по формуле

$$
\mathscr{L}(\alpha)=\min L\left(\alpha_{1}\right) L\left(\alpha_{2}\right) \cdots L\left(\alpha_{m}\right),
$$

где минимум берется по каждому $m \in \mathbb{N}$ и по любым $\alpha_{1}, \alpha_{2}, \ldots, \alpha_{m} \in \overline{\mathbb{Q}}$, удолетворяющим условию $\alpha_{1}+\alpha_{2}+\cdots+\alpha_{m}=\alpha$. Легко видеть, что тогда

$$
\varrho(\alpha, \beta)=\log \mathscr{L}(\alpha-\beta)
$$

определяет расстояние на $\overline{\mathbb{Q}}$. Ясно, что эта метрика индуцирует дискретную топологию, так как расстояние между двумя различными алгебраическими числами будет $\geqslant \log 2$.

Иног да более удобно рассматривать представления $\alpha$ в виде произведения алгебраических чисел, а не их суммы. Для того чтобы определить функцию расстояния, мы вычтем из длины единицу и определим мультипликативный аналог этой метрики на факторгруппе $\overline{\mathbb{Q}}^{*} / \mathscr{U}$, где $\mathscr{U}$ является мультипликативной группой всех корней из единицы степени $2^{m}, m=0,1,2, \ldots$ Определим $\mathscr{L}^{*}(\alpha)$ по формуле

$$
\mathscr{L}^{*}(\alpha)=\min \left(L\left(\alpha_{1}\right)-1\right)\left(L\left(\alpha_{2}\right)-1\right) \cdots\left(L\left(\alpha_{m}\right)-1\right),
$$

где минимум берется по каждому $m \in \mathbb{N}$ и по любым $\alpha_{1}, \alpha_{2}, \ldots, \alpha_{m} \in \overline{\mathbb{Q}}$, удовлетворяюшим условию $\alpha_{1} \alpha_{2} \cdots \alpha_{m}=\alpha$. Тогда

$$
\varrho^{*}(\alpha \mathscr{U}, \beta \mathscr{U})=\log \mathscr{L}^{*}\left(\frac{\alpha}{\beta}\right)
$$

определяет расстояние на $\overline{\mathbb{Q}}^{*} / \mathscr{U}$. (Действительно, неравенство треугольника очевидно, а $L(\alpha)=2$ эквивалентно условию $\alpha \in \mathscr{U}$.) Важным обстоятельством является то, что эта метрика тоже индуцирует дискретную топологию, так как расстояние между двумя различными смежными классами $\alpha \mathscr{U}$ и $\beta \mathscr{U}$, где $\alpha, \beta \neq 0$ и $\alpha / \beta \notin \mathscr{U}$, всегда будет $\geqslant \log 2$.

2. Основные результаты. Очевидно, что $\mathscr{L}(\alpha) \leqslant L(\alpha)$ и $\mathscr{L}^{*}(\alpha) \leqslant L(\alpha)-1$. Насколько малы могут быть величины $\mathscr{L}(\alpha)$ и $\mathscr{L}^{*}(\alpha)$ ? Пример числа $\beta=(-2)^{-1 / d}-1 / N$, где $d \in \mathbb{N}$, а $N$ - большое нечетное положительное число, показывает, что

$$
\mathscr{L}(\beta) \leqslant L\left((-2)^{-1 / d}\right) L\left(-\frac{1}{N}\right)=3(N+1) .
$$

При этом $L(\beta)=2(N+1)^{d}+N^{d}$, так что $\mathscr{L}(\alpha)$ может быть значительно меньше $L(\alpha)$. Следующая теорема дает оценки величин $\mathscr{L}(\alpha)$ и $\mathscr{L}^{*}(\alpha)$.

ТЕОРема 1. Если $\alpha$ - алгебраическое число степени $d$, то

$$
L(\alpha)^{1 / d} \leqslant \mathscr{L}(\alpha) \leqslant L(\alpha), \quad(L(\alpha)-1)^{1 / d} \leqslant \mathscr{L}^{*}(\alpha) \leqslant L(\alpha)-1 .
$$

Kроме того, $\mathscr{L}(\alpha) \geqslant \sqrt{\alpha}+1 u \mathscr{L}^{*}(\alpha) \geqslant \sqrt{\alpha}$.

Через $\alpha$ здесь обозначен максимальный модуль среди всех сопряженных числа $\alpha$ над полем $\mathbb{Q}$.

Как уже замечено вьше, неравенства $\mathscr{L}(\alpha) \leqslant L(\alpha)$ и $\mathscr{L}^{*}(\alpha) \leqslant L(\alpha)-1$ очевидны. Неравенство $\mathscr{L}(\alpha) \geqslant L(\alpha)^{1 / d}$ следует из первой части следующей теоремы. 
Tеорема 2. Если $m \in \mathbb{N}, \alpha_{1}, \alpha_{2}, \ldots, \alpha_{m} \in \overline{\mathbb{Q}} u \alpha=\alpha_{1}+\alpha_{2}+\cdots+\alpha_{m}$ является числом степени $d$, то

$$
L\left(\alpha_{1}+\alpha_{2}+\cdots+\alpha_{m}\right)^{1 / d} \leqslant L\left(\alpha_{1}\right) L\left(\alpha_{2}\right) \cdots L\left(\alpha_{m}\right) .
$$

Также, если $\alpha=\alpha_{1} \alpha_{2} \cdots \alpha_{m}$ является числом степени $d$, то

$$
2^{1-1 / d} L\left(\alpha_{1} \alpha_{2} \cdots \alpha_{m}\right)^{1 / d} \leqslant L\left(\alpha_{1}\right) L\left(\alpha_{2}\right) \cdots L\left(\alpha_{m}\right) .
$$

Доказательства теорем 1 и 2 изложены в п. 4.

Отметим, что если $d=1$, т.е. $\alpha \in \mathbb{Q}$, то из теоремы 1 следует, что $\mathscr{L}(\alpha)=L(\alpha)$ и $\mathscr{L}^{*}(\alpha)=L(\alpha)-1$. При $d=1$ из теоремы 2 получаем такое следствие.

СлЕДСТВИЕ. Если $r \in \mathbb{Q}$ представлено в виде суммы или произведения произвольных алгебраических чисел, то произведение их длин не превышает длины числа $r$.

Интересным является вопрос, насколько точны оценки

$$
\mathscr{L}(\alpha) \geqslant L(\alpha)^{1 / d} \quad \text { и } \quad \mathscr{L}^{*}(\alpha) \geqslant(L(\alpha)-1)^{1 / d} .
$$

Наш пример числа $\beta$ показьвает, что если верно неравенство $\mathscr{L}(\alpha) \geqslant c_{d} L(\alpha)^{1 / d}$, то $c_{d} \leqslant 3^{1-1 / d}$. Более того, если $d$ - степень двойки, то многочлен $x^{d}+1$ неразложим в кольце $\mathbb{Z}[x]$. Для любого из его корней $\zeta \in \mathscr{U}$ верно неравенство $\mathscr{L}(\zeta) \leqslant L(\zeta)=2$. Следовательно, $c_{d} \leqslant 2^{1-1 / d}$, если $d$ - степень двойки. Ясно также, что $\mathscr{L}^{*}(\zeta) \leqslant L(\zeta)-1=1$ и $\mathscr{L}^{*}(\zeta) \geqslant(L(\zeta)-1)^{1 / d}=1$. Таким образом, неравенство $\mathscr{L}^{*}(\alpha) \geqslant(L(\alpha)-1)^{1 / d}$ в теореме 1 неулучшаемо, если $d$ - целая степень двойки. В остальных случаях, пример $\gamma=2^{1 / d}$ показьвает, что если верно неравенство $\mathscr{L}^{*}(\alpha) \geqslant c_{d}^{*}(L(\alpha)-1)^{1 / d}$, то $c_{d}^{*} \leqslant 2^{1-1 / d}$. По-видимому, неравенства $\mathscr{L}(\alpha) \geqslant c_{d} L(\alpha)^{1 / d}$, где $c_{d}=2^{1-1 / d}$ при $d=2^{s}$ и $c_{d}=3^{1-1 / d}$ при $d \neq 2^{s}$, а также $\mathscr{L}^{*}(\alpha) \geqslant c_{d}^{*}(L(\alpha)-1)^{1 / d}$, где $c_{d}^{*}=2^{1-1 / d}$ при $d \neq 2^{s}$, верны.

\section{3. Вспомогательные результаты.}

Лемма 1. Если $\alpha \in \overline{\mathbb{Q}}^{*}$, mo $M(\alpha) \leqslant L(\alpha)-1$.

ДокАЗАТЕЛЬСТво. Пусть $a_{d} x^{d}+\cdots+a_{1} x+a_{0} \in \mathbb{Z}[x]-$ основной многочлен числа $\alpha$. По неравенству Гончалвеса (см., например, [5, с. 244] или [6], где приведен более простой вариант этого неравенства)

$$
\left(M(\alpha)+\left|a_{d} a_{0}\right| M(\alpha)^{-1}\right)^{2}-2\left|a_{d} a_{0}\right|=M(\alpha)^{2}+\left|a_{d} a_{0}\right|^{2} M(\alpha)^{-2} \leqslant\left|a_{d}\right|^{2}+\cdots+\left|a_{1}\right|^{2}+\left|a_{0}\right|^{2} .
$$

Положим $S=\left|a_{d-1}\right|^{2}+\cdots+\left|a_{1}\right|^{2}$. Из неравенства следует, что

$$
M(\alpha) \leqslant \frac{1}{2}\left(\sqrt{S+\left(\left|a_{d}\right|+\left|a_{0}\right|\right)^{2}}+\sqrt{S+\left(\left|a_{d}\right|-\left|a_{0}\right|\right)^{2}}\right) .
$$

Ввиду неравенства $\sqrt{S} \leqslant\left|a_{d-1}\right|+\cdots+\left|a_{1}\right|=L(\alpha)-\left|a_{d}\right|-\left|a_{0}\right|$ легко видеть, что первьй квадратный корень не превышает $L(\alpha)$, а второй не превышает

$$
\sqrt{S}+|| a_{d}|-| a_{0}|| \leqslant L(\alpha)-\left|a_{d}\right|-\left|a_{0}\right|+|| a_{d}|-| a_{0}||=L(\alpha)-2 \min \left\{\left|a_{d}\right|,\left|a_{0}\right|\right\} .
$$

Таким образом,

$$
M(\alpha) \leqslant L(\alpha)-\min \left\{\left|a_{d}\right|,\left|a_{0}\right|\right\} \leqslant L(\alpha)-1 .
$$


Лемма 2. Если $r \in \mathbb{Q} u \alpha \in \overline{\mathbb{Q}}$ имеет степень $d$, mо

$$
L(r+\alpha)^{1 / d} \leqslant L(r) L(\alpha)^{1 / d} \quad u \quad L(r \alpha)^{1 / d} \leqslant M(r) L(\alpha)^{1 / d} .
$$

ДокАЗАТЕЛЬСТво. Ввиду того, что $L(0)=M(0)=1$, неравенства очевидны, если $r=0$ или $\alpha=0$. Пусть $r=u / v \neq 0$, где $u$ и $v$-взаимно простые целые числа. Тогда $L(r)=|u|+|v|$ и $M(r)=\max \{|u|,|v|\}$.

Если $a_{d} x^{d}+\cdots+a_{1} x+a_{0} \in \mathbb{Z}[x]-$ основной многочлен числа $\alpha$, то $u / v+\alpha-$ корень многочлена

$$
Q(x)=a_{d}(v x-u)^{d}+\cdots+a_{1} v^{d-1}(v x-u)+a_{0} v^{d} \in \mathbb{Z}[x] .
$$

Поскольку $\operatorname{deg}(u / v+\alpha)=\operatorname{deg} \alpha=d$, то основным многочленом числа $u / v+\alpha$ будет или сам $Q(x)$, или его делитель степени $d$. Поэтому

$$
\begin{aligned}
L\left(\frac{u}{v}+\alpha\right) & \leqslant L(Q) \leqslant\left|a_{d}\right|(|v|+|u|)^{d}+\cdots+\left|a_{1}\right||v|^{d-1}(|v|+|u|)+\left|a_{0}\right||v|^{d} \\
& \leqslant\left(\left|a_{d}\right|+\cdots+\left|a_{1}\right|+\left|a_{0}\right|\right)(|v|+|u|)^{d}=L(\alpha) L\left(\frac{u}{v}\right)^{d} .
\end{aligned}
$$

Извлекая корень степени $d$, мы получим первое неравенство.

Во втором случае число $u \alpha / v$ является корнем многочлена

$$
R(x)=a_{d} v^{d} x^{d}+\cdots+a_{1} v u^{d-1} x+a_{0} u^{d} \in \mathbb{Z}[x] .
$$

Следовательно, как и вьше,

$$
\begin{aligned}
L\left(\frac{u \alpha}{v}\right) & \leqslant L(R) \leqslant\left|a_{d}\right||v|^{d}+\cdots+\left|a_{1}\right||v||u|^{d-1}+\left|a_{0}\right||u|^{d} \\
& \leqslant\left(\left|a_{d}\right|+\cdots+\left|a_{1}\right|+\left|a_{0}\right|\right) \max \{|u|,|v|\}^{d}=L(\alpha) M\left(\frac{u}{v}\right)^{d} .
\end{aligned}
$$

Извлекая корень степени $d$, мы получим второе неравенство.

Лемма 3. Если $\alpha \in \overline{\mathbb{Q}}$, mo $L(\alpha) \geqslant|\alpha|+1$.

ДокАЗАТЕльство. Если $\alpha$ и $\alpha^{\prime}-$ сопряженные алгебраические числа, т.е. оба являются корнями основного многочлена для $\alpha, a_{d} x^{d}+\cdots+a_{1} x+a_{0} \in \mathbb{Z}[x]$, то $L(\alpha)=L\left(\alpha^{\prime}\right)$. Поэтому достаточно доказать неравенство

$$
L(\alpha) \geqslant|\alpha|+1 .
$$

Оно очевидно при $\alpha=0$ и при $0<|\alpha| \leqslant 1$. Предположим, что $|\alpha|>1$. Из равенства $-a_{d}=a_{d-1} / \alpha+a_{d-2} / \alpha^{2}+\cdots+a_{0} / \alpha^{d}$ следует, что

$$
\left|a_{d}\right| \leqslant\left|\frac{a_{d-1}}{\alpha}\right|+\left|\frac{a_{d-2}}{\alpha^{2}}\right|+\cdots+\left|\frac{a_{0}}{\alpha^{d}}\right| \leqslant \frac{\left|a_{d-1}\right|+\cdots+\left|a_{0}\right|}{|\alpha|} .
$$

Теперь получаем, что

$$
L(\alpha)=\left|a_{d}\right|+\left|a_{d-1}\right|+\cdots+\left|a_{0}\right| \geqslant\left|a_{d}\right|+\left|a_{d}\right||\alpha| \geqslant|\alpha|+1 .
$$

Лемма доказана. 
4. Оценки снизу для произведения длин. Начнем с доказательства неравенства

$$
L\left(\alpha_{1}+\alpha_{2}+\cdots+\alpha_{m}\right)^{1 / d} \leqslant L\left(\alpha_{1}\right) L\left(\alpha_{2}\right) \cdots L\left(\alpha_{m}\right),
$$

где $\alpha=\alpha_{1}+\alpha_{2}+\cdots+\alpha_{m}$ - алгебраическое число степени $d$. Если хотя бы одно из чисел $\alpha_{1}, \alpha_{2}, \ldots, \alpha_{m}$ рационально, то, используя неравенство $L(r+\alpha)^{1 / d} \leqslant L(r) L(\alpha)^{1 / d}$ из леммы 2 , можно применить индукцию по $m$. Поэтому предположим, что $m \geqslant 2$ и $\alpha_{1}, \alpha_{2}, \ldots, \alpha_{m} \in \overline{\mathbb{Q}} \backslash \mathbb{Q}$. Обозначим степени чисел $\alpha_{1}, \alpha_{2}, \ldots, \alpha_{m}$ через $d_{1}, d_{2}, \ldots, d_{m} \geqslant 2$. Используя хорошо известное неравенство $L(\alpha) \leqslant 2^{d} M(\alpha)$, а также неравенство

$$
h\left(\alpha_{1}+\alpha_{2}+\cdots+\alpha_{m}\right) \leqslant h\left(\alpha_{1}\right)+h\left(\alpha_{2}\right)+\cdots+h\left(\alpha_{m}\right)+\log m
$$

(см., например, [7, лемма 3.7]), мы получим, что

$$
\begin{aligned}
L\left(\alpha_{1}+\alpha_{2}+\cdots+\alpha_{m}\right)^{1 / d} & \leqslant 2 M\left(\alpha_{1}+\alpha_{2}+\cdots+\alpha_{m}\right)^{1 / d} \\
& \leqslant 2 m M\left(\alpha_{1}\right)^{1 / d_{1}} M\left(\alpha_{2}\right)^{1 / d_{2}} \cdots M\left(\alpha_{m}\right)^{1 / d_{m}} .
\end{aligned}
$$

Однако по лемме 1 при любом $j=1,2, \ldots, m$

$$
M\left(\alpha_{j}\right)^{1 / d_{j}} \leqslant M\left(\alpha_{j}\right)^{1 / 2} \leqslant\left(L\left(\alpha_{j}\right)-1\right)^{1 / 2} \leqslant \frac{L\left(\alpha_{j}\right)}{2} .
$$

Следовательно,

$$
L\left(\alpha_{1}+\alpha_{2}+\cdots+\alpha_{m}\right)^{1 / d} \leqslant \frac{2 m}{2^{m}} L\left(\alpha_{1}\right) L\left(\alpha_{2}\right) \cdots L\left(\alpha_{m}\right),
$$

что и доказьвает требуемое неравенство, так как $2 m \leqslant 2^{m}$ при $m \geqslant 2$.

Для доказательства неравенства

$$
2^{1-1 / d} L\left(\alpha_{1} \alpha_{2} \cdots \alpha_{m}\right)^{1 / d} \leqslant L\left(\alpha_{1}\right) L\left(\alpha_{2}\right) \cdots L\left(\alpha_{m}\right)
$$

где $\alpha=\alpha_{1} \alpha_{2} \cdots \alpha_{m}$ - алгебраическое число степени $d$, мы сначала заметим, что оно очевидно, если $\alpha=0$ или $m=1$. Если $\alpha \neq 0$, то и все $\alpha_{1}, \alpha_{2}, \ldots, \alpha_{m}$ отличны от нуля. Если хотя бы одно из чисел $\alpha_{1}, \alpha_{2}, \ldots, \alpha_{m}$, например $\alpha_{m}$, рационально, то, применяя леммы 1 и 2 , получим, что

$$
L\left(\alpha_{1} \alpha_{2} \cdots \alpha_{m}\right)^{1 / d} \leqslant M\left(\alpha_{m}\right) L\left(\alpha_{1} \cdots \alpha_{m-1}\right)^{1 / d} \leqslant\left(L\left(\alpha_{m}\right)-1\right) L\left(\alpha_{1} \alpha_{2} \cdots \alpha_{m-1}\right)^{1 / d} .
$$

Следовательно,

$$
2^{1-1 / d} L\left(\alpha_{1} \alpha_{2} \cdots \alpha_{m}\right)^{1 / d}<2^{1-1 / d} L\left(\alpha_{m}\right) L\left(\alpha_{1} \alpha_{2} \cdots \alpha_{m-1}\right)^{1 / d}
$$

и требуемое неравенство легко получается индукцией по $m$. Поэтому предположим, что $m \geqslant 2$, а $\alpha_{1}, \alpha_{2}, \ldots, \alpha_{m}$ - алгебраические числа степеней соответственно $d_{1}, d_{2}, \ldots$, $d_{m} \geqslant 2$. Тогда, пользуясь неравенствами $L(\alpha) \leqslant 2^{d} M(\alpha)$ и

$$
h\left(\alpha_{1} \alpha_{2} \cdots \alpha_{m}\right) \leqslant h\left(\alpha_{1}\right)+h\left(\alpha_{2}\right)+\cdots+h\left(\alpha_{m}\right)
$$


(см. [7, свойство 3.3]), нетрудно убедиться в том, что

$$
L\left(\alpha_{1} \alpha_{2} \cdots \alpha_{m}\right)^{1 / d} \leqslant 2 M\left(\alpha_{1} \alpha_{2} \cdots \alpha_{m}\right)^{1 / d} \leqslant 2 M\left(\alpha_{1}\right)^{1 / d_{1}} M\left(\alpha_{2}\right)^{1 / d_{2}} \cdots M\left(\alpha_{m}\right)^{1 / d_{m}} .
$$

Как и выше, для любого $j=1,2, \ldots, m$

$$
M\left(\alpha_{j}\right)^{1 / d_{j}} \leqslant M\left(\alpha_{j}\right)^{1 / 2} \leqslant\left(L\left(\alpha_{j}\right)-1\right)^{1 / 2},
$$

поэтому

$$
L\left(\alpha_{1} \alpha_{2} \cdots \alpha_{m}\right)^{1 / d} \leqslant 2\left(\left(L\left(\alpha_{1}\right)-1\right)\left(L\left(\alpha_{2}\right)-1\right) \cdots\left(L\left(\alpha_{m}\right)-1\right)\right)^{1 / 2}
$$

при любом $m \geqslant 2$ и при любых $\alpha_{1}, \alpha_{2}, \ldots, \alpha_{m} \in \overline{\mathbb{Q}} \backslash \mathbb{Q}$. Требуемое неравенство теперь следует из оценок

$$
\left(L\left(\alpha_{j}\right)-1\right)^{1 / 2} \leqslant \frac{L\left(\alpha_{j}\right)}{2}
$$

где $j=1,2, \ldots, m, m \geqslant 2$, поскольку

$$
2^{1-1 / d} \cdot 2 \cdot 2^{-m}=2^{2-m-1 / d}<1
$$

Таким образом, теорема 2 доказана. Из первого неравенства теоремы 2 и определения $\mathscr{L}(\alpha)$ мы получаем, что $L(\alpha)^{1 / d} \leqslant \mathscr{L}(\alpha) \leqslant L(\alpha)$. Неравенство $\mathscr{L}^{*}(\alpha) \leqslant L(\alpha)-1$ очевидно. Так как $\mathscr{L}^{*}(\alpha \zeta)=\mathscr{L}^{*}(\alpha)$ при любом $\zeta \in \mathscr{U}$, то для доказательства неравенства $\mathscr{L}^{*}(\alpha) \geqslant(L(\alpha)-1)^{1 / d}$ достаточно показать, что

$$
\left(L\left(\alpha_{1} \alpha_{2} \cdots \alpha_{m}\right)-1\right)^{1 / d} \leqslant\left(L\left(\alpha_{1}\right)-1\right)\left(L\left(\alpha_{2}\right)-1\right) \cdots\left(L\left(\alpha_{m}\right)-1\right)
$$

при любых $\alpha_{1}, \alpha_{2}, \ldots, \alpha_{m} \notin \mathscr{U}$. Здесь $\alpha=\alpha_{1} \alpha_{2} \cdots \alpha_{m}$ - алгебраическое число степени $d$. Это неравенство очевидно, если $\alpha=0$ или $m=1$. Более того, как и выше, не ограничивая общности, можно считать, что все $\alpha_{1}, \alpha_{2}, \ldots, \alpha_{m}$ иррациональны, ибо при $\alpha_{m} \in \mathbb{Q}$

$$
\left(L\left(\alpha_{1} \alpha_{2} \cdots \alpha_{m}\right)-1\right)^{1 / d}<L\left(\alpha_{1} \alpha_{2} \cdots \alpha_{m}\right)^{1 / d} \leqslant\left(L\left(\alpha_{m}\right)-1\right) L\left(\alpha_{1} \alpha_{2} \cdots \alpha_{m-1}\right)^{1 / d} .
$$

Однако при этих условиях нами уже получено неравенство $L\left(\alpha_{1} \alpha_{2} \cdots \alpha_{m}\right)^{1 / d} \leqslant$ $2\left(\left(L\left(\alpha_{1}\right)-1\right) \cdots\left(L\left(\alpha_{m}\right)-1\right)\right)^{1 / 2}$. Так как $\alpha_{j} \notin \mathscr{U} \cup\{0\}$, то $L\left(\alpha_{j}\right) \geqslant 3$ для каждого $j=1,2, \ldots, m$. Следовательно, $\left(L\left(\alpha_{j}\right)-1\right)^{1 / 2} \leqslant\left(L\left(\alpha_{j}\right)-1\right) / \sqrt{2}$ и

$$
\left(L\left(\alpha_{1} \alpha_{2} \cdots \alpha_{m}\right)-1\right)^{1 / d}<L\left(\alpha_{1} \alpha_{2} \cdots \alpha_{m}\right)^{1 / d} \leqslant 2^{1-m / 2}\left(L\left(\alpha_{1}\right)-1\right) \cdots\left(L\left(\alpha_{m}\right)-1\right) .
$$

Это доказьвает требуемое неравенство, поскольку $m \geqslant 2$.

Для завершения доказательства теоремы 1 остается показать, что $\mathscr{L}(\alpha) \geqslant|\alpha|+1$ и $\mathscr{L}^{*}(\alpha) \geqslant \propto$. Если $\mathscr{L}(\alpha)=L\left(\alpha_{1}\right) L\left(\alpha_{2}\right) \cdots L\left(\alpha_{m}\right)$, где $\alpha=\alpha_{1}+\alpha_{2}+\cdots+\alpha_{m}$, то из того, что любое сопряженное числа $\alpha$, скажем $\alpha^{\prime}$, можно записать как сумму чисел сопряженных соответственно к $\alpha_{1}, \alpha_{2}, \ldots, \alpha_{m}$, следует равенство $\mathscr{L}(\alpha)=\mathscr{L}\left(\alpha^{\prime}\right)$. Аналогично, $\mathscr{L}^{*}(\alpha)=\mathscr{L}^{*}\left(\alpha^{\prime}\right)$.

Поэтому для первого неравенства достаточно доказать, что $\mathscr{L}(\alpha) \geqslant|\alpha|+1$. Для этого мы воспользуемся леммой 3 и представлением $\alpha=\alpha_{1}+\alpha_{2}+\cdots+\alpha_{m}$. Тогда

$$
\begin{aligned}
\mathscr{L}(\alpha) & =L\left(\alpha_{1}\right) L\left(\alpha_{2}\right) \cdots L\left(\alpha_{m}\right) \geqslant\left(\left|\alpha_{1}\right|+1\right)\left(\left|\alpha_{2}\right|+1\right) \cdots\left(\left|\alpha_{m}\right|+1\right) \\
& \geqslant\left|\alpha_{1}+\alpha_{2}+\cdots+\alpha_{m}\right|+1=|\alpha|+1 .
\end{aligned}
$$

Неравенство $\mathscr{L}^{*}(\alpha) \geqslant \mid \alpha$ следует из неравенства $\mathscr{L}^{*}(\alpha) \geqslant|\alpha|$, которое получается аналогичным образом: если $\mathscr{L}^{*}(\alpha)=\left(L\left(\alpha_{1}\right)-1\right)\left(L\left(\alpha_{2}\right)-1\right) \cdots\left(L\left(\alpha_{m}\right)-1\right)$, где $\alpha=$ $\alpha_{1} \alpha_{2} \cdots \alpha_{m}$, то

$$
\mathscr{L}^{*}(\alpha)=\left(L\left(\alpha_{1}\right)-1\right)\left(L\left(\alpha_{2}\right)-1\right) \cdots\left(L\left(\alpha_{m}\right)-1\right) \geqslant\left|\alpha_{1}\right|\left|\alpha_{2}\right| \cdots\left|\alpha_{m}\right|=|\alpha| .
$$




\section{СПИСОК ЦИТИРОВАННОЙ ЛИТЕРАТУРЫ}

[1] Dubickas A., Smyth C. J. On the metric Mahler measure // J. Number Theory. 2001. V. 86. P. $368-387$.

[2] Dubickas A., Smyth C. J. On metric heights // Period. Math. Hungar. 2003. V. 42. P. 135-155.

[3] Schmidt W. Heights of points on subvarieties of $\mathbb{G}_{m}^{n} / /$ Contemp. Math. 1998. V. 210. P. 97-99.

[4] Dubickas A. Asymptotic density of surds with stable height // Acta Appl. Math. 2003. V. 78. P. 99-102.

[5] Schinzel A. Polynomials with Special Regard to Reducibility. Cambridge: Cambridge Univ. Press, 2000.

[6] Фельдман Н. И. Приближения алгебраических чисел. М.: Изд-во Московского ун-та, 1981.

[7] Waldschmidt M. Diophantine Approximation on Linear Algebraic Groups. Berlin-New York: Springer-Verlag, 2000.

(А. Дубицкас) Вильнюсский университет, Литва

Поступило

(К. Смит) Университет Эдинбурга, Великобритания

02.04 .2004

E-mail: arturas.dubickas@maf.vu.1t, chris@maths.ed.ac.uk 